\title{
Method of Phase Diagrams for the Analysis of Seism-Acoustical Spatial-Time Monitoring Data in Oil Wells
}

\author{
Olga Hachay ${ }^{1}$, Oleg Khachay ${ }^{2}$ \\ ${ }^{1}$ Institute of Geophysics, Ural Branch of Russian Academy of Sciences, Yekaterinburg, Russia \\ ${ }^{2}$ Ural Federal University, Yekaterinburg, Russia \\ Email: olgakhachay@yandex.ru, khachay@yandex.ru
}

How to cite this paper: Hachay, $\mathrm{O}$. and Khachay, O. (2018) Method of Phase Diagrams for the Analysis of Seism-Acoustical Spatial-Time Monitoring Data in Oil Wells. Open Journal of Geology, 8, 874-882. https://doi.org/10.4236/ojg.2018.89051

Received: July 19, 2018

Accepted: August 3, 2018

Published: August 6, 2018

Copyright (c) 2018 by authors and Scientific Research Publishing Inc. This work is licensed under the Creative Commons Attribution-NonCommercial International License (CC BY-NC 4.0).

http://creativecommons.org/licenses/by-nc/4.0/

\begin{abstract}
Experimental and theoretical studies of the mechanisms of vibration stimulation of oil recovery in watered fields lead to the conclusion that resonance oscillations develop in fractured-block formations. These oscillations, caused by weak but long-lasting and frequency-stable influences, create the conditions for ultrasonic wave's generation in the layers, which are capable of destroying thickened oil membranes in reservoir cracks. For fractured-porous reservoirs in the process of exploitation by the method of water high-pressure oil displacement, the possibility of intensifying ultrasonic vibrations can have an important technological significance. Even a very weak ultrasound can destroy, over a long period of time, the viscous oil membranes formed in the cracks between the blocks, which can be the reason for lowering the permeability of the layers and increasing the oil recovery. To describe these effects, it is necessary to consider the wave process in a hierarchically blocky environment and theoretically simulate the mechanism of the appearance of self-oscillations under the action of relaxation shear stresses. For the analysis of seism acoustic response in time on fixed intervals along the borehole an algorithm of phase diagrams of the state of many-phase medium is suggested.
\end{abstract}

\section{Keywords}

Phase Diagrams, Method of Analysis, Space-Time Monitoring Data, Oil Wells, State of the Two Component Medium

\section{Introduction}

The phenomenon of the Institute of Geophysics of the Ural Branch of the Russian Academy of Sciences is developing and improving the method of active 
seism acoustic monitoring of the oil reservoir [1] [2] [3] [4] [5]. This method is used to assess the oil saturation of the formation and its ability to oil recovery.

An empirical fact is established about the influence of the vibration effect on the acceleration of oil recovery processes. A physical explanation for this can be given by the following model. The active medium has its high energy and often a non-equilibrium structural state. Therefore, it is necessary to expect synchronizing or synergetic action on the meso-processes in the zones of maximum instability, reduced strength and high fatigue of the medium at the meso-level from the influencing signal. At these levels, the energy of the impact can accumulate in the meso-structures and cause mechanical effects that initiate mechanical movements and fragmentation of the medium [6] [7] [8].

At present, there are many publications in the domestic and foreign literature devoted to the physical-mathematical model of the process of seism acoustic radiation caused by the action of a fixed frequency on the medium. That model is used for the theoretical justification of seismic-acoustic monitoring of the reservoir condition before and after the seismic impact on it.

It is known that in an ideal elastic medium, i.e. in the absence of boundaries on which the displacement field or stress field breaks, energy cannot accumulate over time and remain after the source is turned off; therefore such a continuous medium can not correspond to the observed phenomena of the formation and relaxation of seismic emission with the presence of natural frequencies of the medium. In block structures, as Academician M.A. Sadovsky has shown, and also in a hierarchically blocked environment, it is possible to form secondary emissions of seismic waves with dominant "natural frequencies" and after prolonged exposure to such medium, interblock contact zones are weakened and dynamic fragmentation is amplified.

Consider the following experimentally observed fact-an increase in oil recovery under the influence of dynamic restructuring of the block energy-saturated oil deposit. With the long-term exploitation of the deposit, the skeleton base of the oil reservoir is gradually destroyed, which leads to a significant increase in internal stresses and weakening of the bonds between the constituent individual blocks of reservoirs. In this case, even a small impulse can lead to a restructuring of the reservoir structure, increase the mobility of oil and increase its part in the borehole fluid [9] [10].

Experimental and theoretical studies of the mechanisms of vibration stimulation of oil recovery in watered fields lead to the conclusion that resonance oscillations develop in fractured-block formations. These oscillations, caused by weak but long-lasting and frequency-stable influences, create the conditions for ultrasonic wave's generation in the layers, which are capable of destroying thickened oil membranes in reservoir cracks.

For fractured-porous reservoirs in the process of exploitation by the method of water high-pressure oil displacement, the possibility of intensifying ultrasonic vibrations can have an important technological significance. Even a very weak 
ultrasound can destroy, over a long period of time, the viscous oil membranes formed in the cracks between the blocks, which can be the reason for lowering the permeability of the layers and increasing the oil recovery [11].

To describe these effects, it is necessary to consider the wave process in a hierarchically blocky environment and theoretically simulate the mechanism of the appearance of self-oscillations under the action of relaxation shear stresses [12].

\section{Oil Reservoir as an Open Dynamical System}

On the other hand, the oil reservoir under periodic vibration is an open dynamic system. A dynamical system is understood as an object or process for which the concept of state as a collection of values of certain quantities at a given instant of time is defined, and an operator defining the evolution of the initial state in time is defined [13]. In what follows we shall use the information of the theory of dynamical systems from the monograph [13]. If to describe the behavior of a system it is sufficient to know its state at a finite number of instants of time, then such a system is called a system with discrete time. Let the set of numbers $\mathrm{x}=\left\{X_{1}\right.$, $\left.x_{2}, \cdots, x_{N}\right\}$ describe the state of the dynamical system at a certain instant of time and different states correspond to different sets $\left\{x_{1}, x_{2}, \cdots, x_{N}\right\}$. We define an evolution operator, indicating the rate of change of each state of the system

$$
\frac{\partial x_{i}}{\partial t}=F_{i}\left(t, x_{1}, x_{2}, \cdots, x_{N}\right), i=1, \cdots, N
$$

$\boldsymbol{x}$ is a point of the Euclidean space $\mathrm{P}_{\mathrm{N}}$, which is called a phase space, and $\boldsymbol{x}$ is a phase point. A system of the form (1), in which the right-hand side does not depend on time, is called autonomous. When studying the dynamic system associated with a change in the state of the oil reservoir due to vibration action, the right-hand sides of equations (1) will depend on time, and system (1) will not be autonomous. If the system of equations is supplemented by the initial conditions $x(0)=x_{0}$, then we obtain the initial problem (the Cauchy problem) for the system of Equations (1). Its solution $\{\boldsymbol{x}(t), t>0\}$, considered as the set of points of the phase space $\mathrm{P}_{\mathrm{N}}$, forms a phase trajectory; the vector-valued function $F(\boldsymbol{x})$ defines a vector velocity field. The phase trajectories and the vector velocity field give a visual representation of the behavior of the system over time. The set of phase trajectories corresponding to different initial conditions form a phase portrait of the dynamical system.

Dynamic systems are divided into conservative and dissipative systems. For the former, the total energy of the system is preserved, for the second, energy losses are possible. As to our task of studying the state of an array that is in the process of working out, the model of an inhomogeneous and non-stationary dissipative system is closest. Nevertheless, in the array can be such local parts of it, which will be described by a conservative dynamic model, i.e. by model of energy balance.

The analysis of the phase portrait of the dynamic system allows drawing a conclusion about the state of the system for the period of its observation. So, in 
conservative systems there are no attracting sets. An attractor is a subset of the phase space $\mathrm{P}_{\mathrm{N}}$, to which trajectories tend with time starting in some neighborhood of it. If in a conservative system there is a periodic motion, then such motions are infinitely many and are determined by the initial value of the energy. Attractive sets can exist in dissipative systems. Stationary oscillations for dissipative dynamical systems are not characteristic. However, in nonlinear systems it is possible to have a periodic asymptotically stable motion, the mathematical image of which is the limit cycle, represented in phase space by a closed line, to which trajectories from some neighborhood of this line are contracted with time.

In terms of the shape of the phase portrait, one can judge the characteristic behavior of the system, and the "smooth" deformations of the phase space do not lead to qualitative changes in the dynamics of the system. This property is called the topological equivalence of phase portraits. It allows you to analyze the behavior of various dynamic systems from a single point of view: on its basis, the set of dynamical systems under consideration can be divided into classes within which systems demonstrate qualitatively similar behavior. From the mathematical point of view, the "smooth deformation" of the phase portrait is a one-to-one and mutually continuous transformation of the phase coordinates, as a result of which new singular points cannot appear, and on the other hand-singular points cannot disappear.

\section{Algorithm for Constructing Phase Diagrams of the Two Component Medium State}

Let us consider the algorithm for constructing a phase portrait or diagram from seism acoustic monitoring data. As a result of the well monitoring, we have three sets of intensity data of the seism acoustic radiation: background $I(t, z)_{f}$, after the first excitation $I(t, z)_{V 1}$ and after the second excitation $I(t, z)_{V 2}$. These three functions for a fixed $z$ are observed for a time interval of 14 seconds with a sampling frequency of $44,100 \mathrm{~Hz}$ with a borehole step of $0.5 \mathrm{~m}$. The entire interval is divided into 14 sub-intervals of $1 \mathrm{sec}$ duration. Within each second, the observed values are averaged $I(t, z)_{f}, I(t, z)_{V 1}, I(t, z)_{V 2}$ over the intervals: 0 - $0.1 \mathrm{sec}, 0.1-0.2 \mathrm{sec}, 0.2-0.3 \mathrm{sec}, 0.3-04 \mathrm{sec}, 0.4-0.5 \mathrm{sec}, 0.5-0.6 \mathrm{sec}, 0.6-0.7$ sec, $0.7-0.8 \mathrm{sec}, 0.8-0.9 \mathrm{sec}, 0.9-1 \mathrm{sec}$. Thus, we obtain a new database of averaged data $I_{s}(t, z)_{f}, I_{s}(t, z)_{V 1}, I_{s}(t, z)_{V 2}$, for which we construct phase diagrams on the planes

$$
I_{s}(t, z)_{f}, \frac{\partial}{\partial t} I_{s}(t, z)_{f} ; I_{s}(t, z)_{V 1}, \frac{\partial}{\partial t} I_{s}(t, z)_{V 1} ; I_{s}(t, z)_{V 2}, \frac{\partial}{\partial t} I_{s}(t, z)_{V 2} ;
$$

Then we determine: $\max I_{s}(t, z)_{f}, \min I_{s}(t, z)_{f}, \max \left|\frac{\partial}{\partial t} I_{s}(t, z)_{f}\right|$. Similarly for the functions $I_{s}(t, z)_{V 1}$ and $I_{s}(t, z)_{V 2}$. After that, we calculate the areas of three figures (rectangles that include phase portraits of the three sets of functions (2)), for example: 


$$
\begin{aligned}
A_{f}= & \left(\max I_{s}(t, z)_{f}-\min I_{s}(t, z)_{f}\right) \\
& \times\left(\max \left|\frac{\partial}{\partial t} I_{s}(t, z)_{f}\right|, \frac{\partial}{\partial t}>0+\max \left|\frac{\partial}{\partial t} I_{s}(t, z)_{f}\right|, \frac{\partial}{\partial t} \leq 0\right)
\end{aligned}
$$

The quantity (3) for each time interval, equal to $1 \mathrm{sec}$, is denoted $A_{f}, A_{V 1}, A_{V 2}$ respectively. Next, we construct the phase diagrams on the planes:

$$
A_{f}, \frac{\partial A_{f}}{\partial t}, A_{V 1}, \frac{\partial A_{V 1}}{\partial t}, A_{V 2}, \frac{\partial A_{V 2}}{\partial t}
$$

which will be further analyzed to describe the state of the near-well oil-saturated reservoir. This algorithm is realized in Microsoft Excel Visual Basic in the form of macros that allows quickly average data with an arbitrary frequency and calculate the described quantities.

\section{Examples of Processing Seism Acoustic Monitoring Data, Using the Method of Phase Diagrams}

Here on the Figures 1(a)-(c) we can see how the morphology of phase diagrams changes during the acoustic influence of the reservoir medium in the oil wells.

From the processing results described by the algorithm of full-scale data it follows that the background phase diagrams before excitations vary greatly in their morphology, by attracting point and the area under the phase trajectory for each observation point along the well, indicating a sharp heterogeneity of the wellbore environment. Phase diagrams before and after the first and second impacts at the same point along the well differ by the same criteria, reflecting the fact that the well near the wellbore changes its state with vibration. If the area under the phase diagram after the first influence becomes larger than the analogous value before the influence and, or the value $A_{V 1}^{c}=\left(\max A_{V 1}-\min A_{V 1}\right)$ becomes larger than $A_{f}^{c}=\left(\max A_{f}-\min A_{f}\right)$, this means that the intensity of the seism acoustic radiation has also increased, which indicates the closure of cracks and pores and the decrease of the influence of the liquid phase. If these parameters become correspondingly smaller, this means that the intensity of the seism acoustic radiation has decreased, which indicates a larger flow of the liquid phase and an increase its mobility in a given time interval and at a given observation point. A similar analysis is made relative to the phase diagram after the second excitation.

\section{Conclusions}

At the present time, using the seism acoustic monitoring data of a large number of wells in the West Siberia field, whose oil interval is located in the same geological formation and at a relatively same depth, the quantitative oil saturation and oil mobility tests are developed using the proposed algorithm to form a training sample and the subsequent use of the method of pattern recognition to solve practical problems.

Thus, the developed method of analyzing seism acoustic information allows 


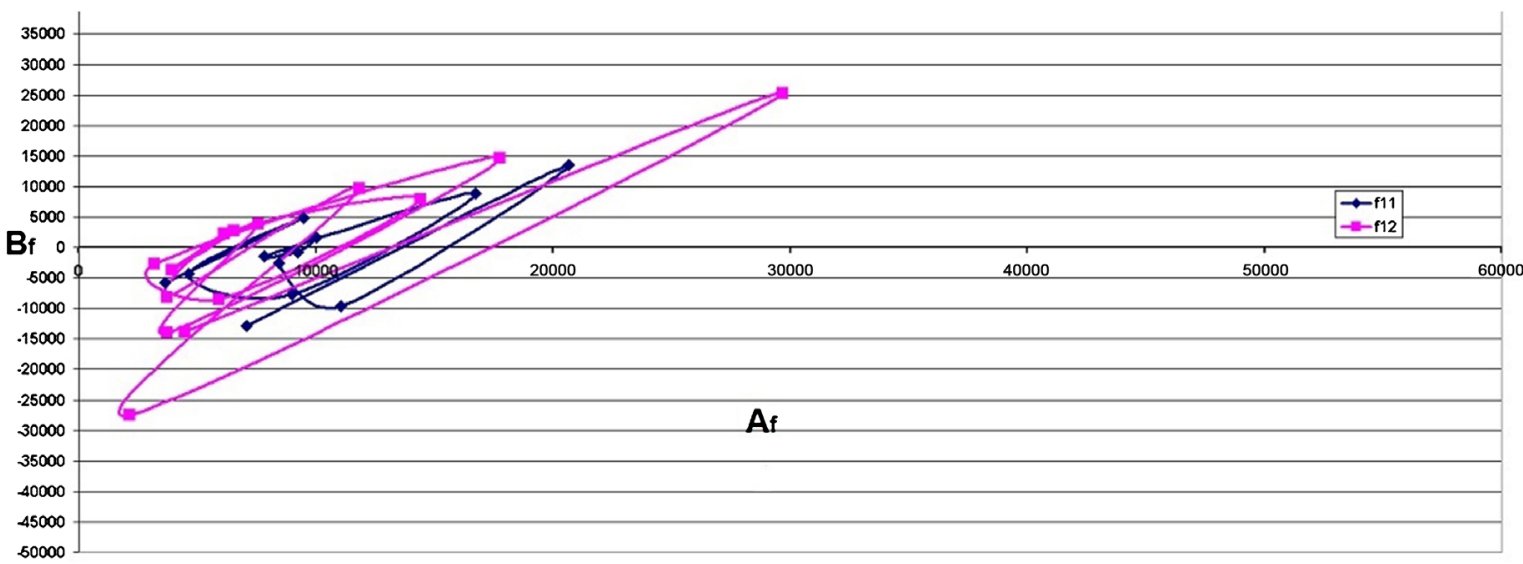

(a)

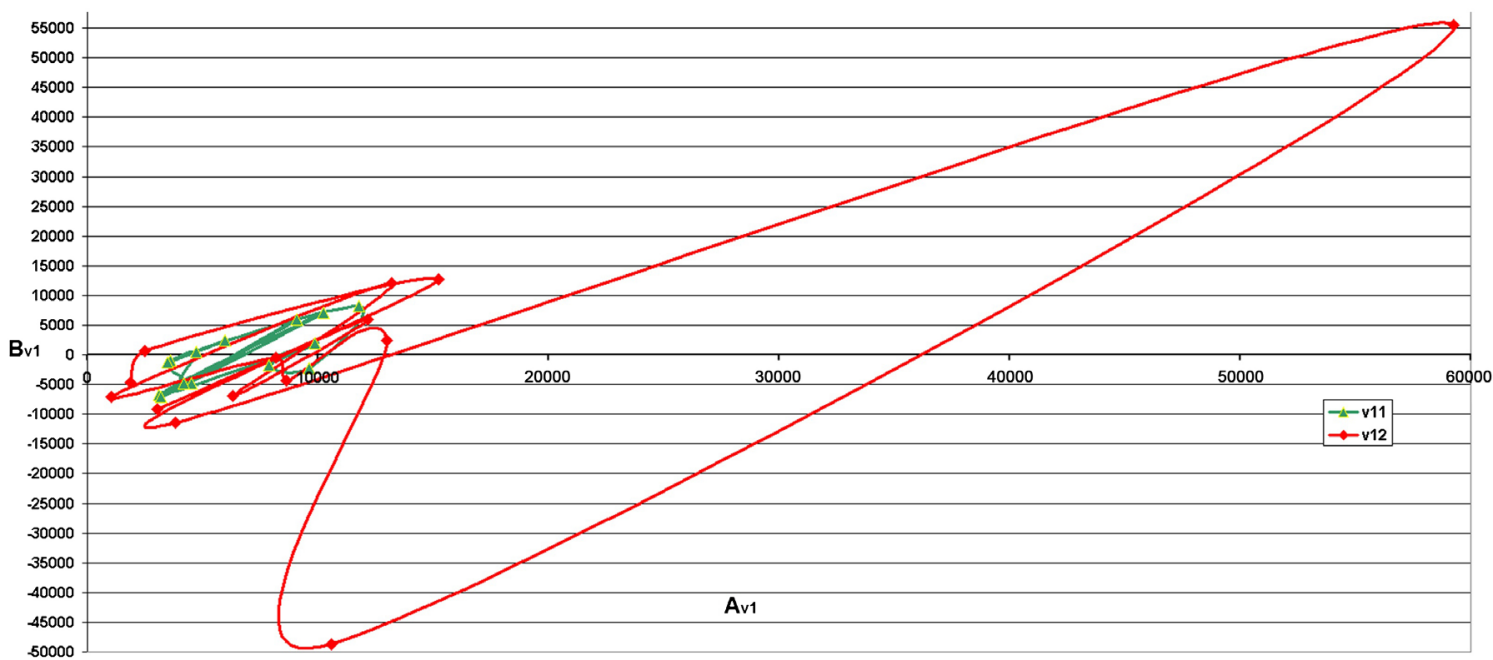

(b)

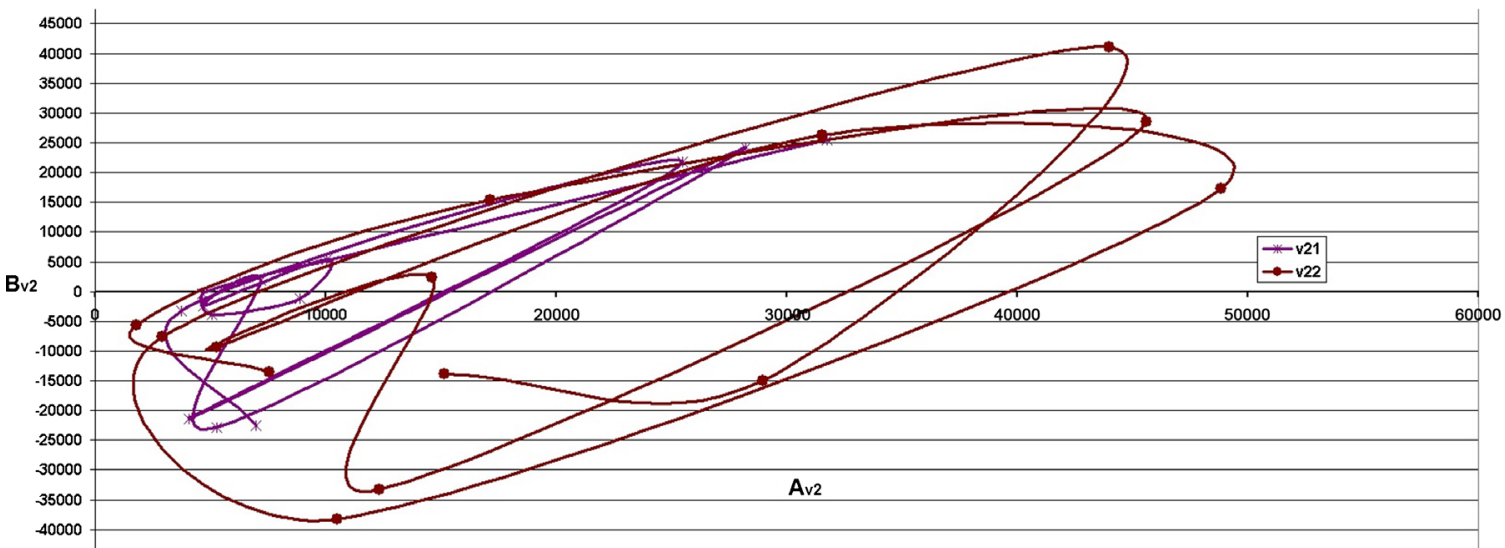

(c)

Figure 1. Phase diagrams of one point of the oil well, depth $2626 \mathrm{~m}$ : (a) before the influence $A_{f}, B_{f}=\frac{\partial A_{f}}{\partial t}$, f11-at the beginning of the cycle, f12-at the end of the cycle; (b) after one cycle of frequency acoustic influence $A_{v 1}, B_{v 1}=\frac{\partial A_{v 1}}{\partial t}$ v11-at the beginning of the cycle, v12-at the end of the cycle; (c) after the second cycle of frequency acoustic influence $A_{v 2}, B_{v 2}=\frac{\partial A_{v 2}}{\partial t}$ v21-at the beginning of the cycle, v22-at the end of the cycle. 
qualitatively classifying the state of a multiphase medium, which is the oil reservoir, using data from multiple impacts. For the quantitative solution of the above-mentioned phenomena of the non-equilibrium and hysteresis interaction of water and oil during the development of an oil reservoir, it is necessary to supplement and develop a system of seism acoustic observations.

Let us consider non-equilibrium models of two-phase filtration. The basis of modern calculations of two-phase filtration flows is the phenomenological theory of the Muskete-Leveretta [14] based on the equations for the conservation of phase masses and the generalized Darcy law for phase flows and is closed by empirical functions of phase permeabilities and capillary pressure. It is the kind of phase permeability functions that determine the concrete picture of a two-phase flow (in particular, the process of displacement of oil by water).

In general theoretical constructions, knowledge of the qualitative properties of the phase permeability functions and capillary pressure is sufficient. When carrying out calculations of specific objects, they must be determined experimentally, on a sufficiently representative (taking into account the heterogeneity of the formation) material and with sufficient consideration of the sensitivity of the results to the form of phase permeabilities-accuracy.

The most common way to determine the functions of relative phase permeabilities (RPT) is to conduct laboratory tests on cores. Experimental determination of the dependence of the RPT on water saturation is a laborious process requiring the use of special equipment. Therefore, in practice, the ready-made dependences of the RPT obtained for rocks of a similar type or for the simplest porous media, such as samples from bulk sand, are often used. This approach is not always valid, since the PFD, even for the same class of rocks, is determined by a large number of factors. There are data [15] proving that when using in the hydrodynamic calculations the parameters of the development of an oil field for the RPT curves obtained not for the conditions of a given deposit, errors in the results and, above all, in the dynamics of water cut, can reach $60 \%-80 \%$.

With the introduction of a large number of oil and gas fields, characterized by a variety of geological and geophysical properties, the need for reliable data on the phase permeabilities of a particular deposit increases. In practice, the extrusion method is widely used to determine the RPT, in which water displacing oil is pumped into a sample of a porous medium saturated with oil and bound water. The results of laboratory studies [16] and numerical simulation [17] have shown that the curves of the phase transition of a two-phase system are not constant in time, which indicates a non-equilibrium character of the immiscible displacement.

According to modern ideas, the non-equilibrium phase permeabilities are associated with the processes of transfer between pores. In this connection, experiments that establish the character of the distribution of residual oil after displacement by water are of particular interest. It was shown [18] that residual oil globules have dimensions of $\sim 1-10$ pore diameters. With a rapid change in the 
displacement regime [18], when the characteristic time of regime change is less than the characteristic time of displacement from the capillaries, the phase permeabilities of both displaced and displacing phases are less than in the equilibrium case, and also with non-equilibrium displacement, the residual saturation depends on the viscosity of the displacing and displaced phases and increases with an increase in the degree of non-equilibrium.

The classical models are based on the idea of a unique dependence of the phase permeability functions and capillary pressure on saturation, which physically means a unique relationship between phase distribution in pores and saturation. This assumption is true if the porous medium is macro homogeneous at distances sufficiently large, including a wide range of pore sizes. However, real oil and gas reservoirs have heterogeneity in terms of porosity and permeability of the most varied scale. Therefore, classical models of two-phase filtration can be considered as an equilibrium approximation to the description of the process. Oil recovery in a heterogeneous reservoir does not depend monotonically on the rate of displacement, which is explained by the finite time of establishing the equilibrium distribution of the phases. We must move on to models with hierarchical inclusions in a layered-block environment.

\section{Acknowledgements}

The work was carried out according to the Program of Fundamental Research of the Department of Earth Sciences of the Russian Academy of Sciences "Fundamental problems of geology, sedimentology, geochemistry of oil and gas, development of new technologies for forecasting, prospecting, exploration and development of traditional and non-traditional hydrocarbon fields".

Authors are thankful to V.V. Dryagin for the seism acoustic monitoring data that we used in our paper as examples for processing.

\section{Conflicts of Interest}

The authors declare no conflicts of interest regarding the publication of this paper.

\section{References}

[1] Dryagin, V.V., Igolkina, G.V. and Ivanov, D. (2009) Experience of Studying the Informative Characteristics of Induced Acoustic Emission in Oil-Saturated Reservoirs of a Terrigenous Type. Proceedings of the International Conference. Geodynamics. Deep Structure. The Thermal Field of the Earth. Interpretation of Geophysical Fields. Fifth Scientific Readings Yu.P. Bulashevitch. IGF UB RAS, Ekaterinburg, 168-174.

[2] Hachay, O.A. and Dryagin, V.V. (2010) Method of Phase Diagrams for the Analysis of Seism-Acoustic Space-Time Monitoring of Oil Wells. Problems of Theory and Practice of Geological Interpretation of Gravitational, Magnetic and Electric Fields. Proceedings of the 37-th Session of the International Seminar. D.G. Uspensky. Moscow IFZ RAS, 380-385.

[3] Hachay, O.A. and Dryagin, V.V. (2010) Analysis of Seism-Acoustic Active Spa- 
tial-Temporal Monitoring of the Geological Environment from the Position of an Open Dynamic System. Proceedings of the Scientific Conference Devoted to the 100 th Anniversary of the Birth of A.V. Rimsky-Korsakov, Moscow AKIN, RAO, 143-146.

[4] Dryagin, V., Ivanov, D., Nigmatullin, D. and Shumilov, A. (2014) Seism Acoustic Emission of a Productive Formation in the Technology of Detection and Extraction of Hydrocarbons. Geophysics, 4, 54-59.

[5] Dryagin, V. (2013) Seism Acoustic Emission of Oil Reservoir. Acoustic Journal, 59, 744-751. (In Russian)

[6] Lavrov, A.V. and Shkuratnik, V.L. (2005) Acoustic Emission during Deformation and Fracture of Rocks (Review). Acoustic Journal, 51, Appendix, 6-18.

[7] Chebotareva, I., Volodin, I. and Dryagin, V. (2017) Acoustic Effects in the Deformation of Structurally Heterogeneous Media. Acoustic Journal, 63, 84-93.

[8] Chebotareva, I., Volodin, I. and Dryagin, V. (2016) Generation of a Low-Frequency Branch of Acoustic Emission in Rocks under the Influence. Doklady Earth Sciences, 468, 205-208. https://doi.org/10.1134/S1028334X16050160

[9] Mitrofanov, V.P., Dzyubenko, A.I., Nechaeva, N.Yu. and Dryagin, V.V. (1998) Results of Field Trials of Acoustic Impact on the Bottom Hole Formation Zone. Geology, Geophysics and Development of Oil Fields, 10, 29-35.

[10] Volodin, I. and Chebotareva, I. (2014) Seismic Emission in Zones of Technogenic Impacts. Acoustic Journal, 60, 505-517.

[11] Alekseev, A.S., Tsetsokho, V.A., Belonosov, A.V. and Skazka, V.V. (2001) Forced Vibrations of Fractured-Block Fluid-Saturated Layers under Vibro Seismic Influences. Physical and Technical Problems of Mining, 6, 3-12.

[12] Hachay, O.A. and Khachay, O.Yu. (2008) Modeling of the Seismic and Electromagnetic Field in Hierarchically Heterogeneous Media. Geophysical Studies of the Urals and Adjacent Regions. Proceedings of the International Conference. IGF UB RAS, Ekaterinburg, 295-299.

[13] Chulichkov, A.I. (2003) Mathematical Models of Nonlinear Dynamics. Phizmatlit, Moscow.

[14] Martos, V.N. and Ryzhik, V.M. (1972) Determination of the Dynamic Curves of Capillary Pressure by the Stabilized Zone Method. Fluid Dynamics, 7, 57-60. https://doi.org/10.1007/BF01186481

[15] Entov, V.M. and Cheng-Shin, E. (1987) Micromechanics of Two-Phase Flow in Porous Media. Numerical Methods for Solving Filtration Problems for a Multiphase Incompressible Fluid. Institute of Theoretical and Applied Mechanics, Novosibirsk, 120-129.

[16] Mats, A.A. and Polischuk, A.M. (1983) Visualization of Oil Remaining in a Porous Medium after Water Flooding. In: Mathematical Methods in Problems of Petrophysics and Correlation, Science, Moscow, 125-130.

[17] Ryzhik, V.M. (1964) Oil Displacement by Water in a Porous Medium with Low Permeability Inclusions. Izvestia of AN USSR Mechanics of Fluid and Gas, 1, 126-132.

[18] Khasanov, M.M. and Bulgakova, G.T. (2003) Nonlinear and Non-Eqiulibrium Effects in Rheological Complex Environments.-Moscow. Institute for Computer Research, Izhevsk. 